\title{
Easily identified at-risk patients for extubation failure may benefit from noninvasive ventilation: a prospective before-after study
}

Arnaud W. Thille ${ }^{1,2,3,4^{*}}$, Florence Boissier ${ }^{1,2,3,4+}$, Hassen Ben-Ghezala ${ }^{4+}$, Keyvan Razazi ${ }^{4}$, Armand Mekontso-Dessap ${ }^{4}$, Christian Brun-Buisson ${ }^{4}$ and Laurent Brochard ${ }^{5,6}$

\begin{abstract}
Background: While studies have suggested that prophylactic noninvasive ventilation (NIV) could prevent postextubation respiratory failure in the intensive care unit, they appear inconsistent with regard to reintubation. We assessed the impact of a prophylactic NIV protocol on reintubation in a large population of at-risk patients.

Methods: Prospective before-after study performed in the medical ICU of a teaching referral hospital. In the control cohort, we determined that patients older than 65 years and those with underlying cardiac or respiratory disease were at high-risk for reintubation. In the interventional cohort, we implemented a protocol using prophylactic NIV in all patients intubated at least $24 \mathrm{~h}$ and having one of these risk factors. NIV was immediately applied after planned extubation during at least the first 24 hours. Extubation failure was defined by the need for reintubation within seven days following extubation.

Results: We included 83 patients at high-risk among 132 extubated patients in the control cohort (12-month period) and 150 patients at high-risk among 225 extubated patients in the NIV cohort (18-month period). The reintubation rate was significantly decreased from $28 \%$ in the control cohort (23/83) to $15 \%(23 / 150)$ in the NIV cohort $(p=0.02$ log-rank test), whereas the non-at-risk patients did not significantly differ in the two periods $(10.2 \%$ vs. $10.7 \%$, $p=0.93$ ). After multivariate logistic-regression analysis, the use of prophylactic NIV protocol was independently associated with extubation success.
\end{abstract}

Conclusions: The implementation of prophylactic NIV after extubation may reduce the reintubation rate in a large population of patients with easily identified risk factors for extubation failure.

\section{Background}

Extubation is a critical decision in an ICU and extubation failure is associated with high mortality $[1,2]$. Despite having successfully passed a weaning readiness test, $15 \%$ of patients on average and up to $20-25 \%$ of those at highrisk may need reintubation [3-6].

Several studies have suggested that prophylactic noninvasive ventilation (NIV) could help to prevent postextubation respiratory failure in patients at high-risk for

\footnotetext{
* Correspondence: aw.thille@gmail.com

${ }^{\dagger}$ Equal contributors

${ }^{1} \mathrm{CHU}$ de Poitiers, Réanimation Médicale, Poitiers, France

${ }^{2}$ INSERM CIC 1402 (ALIVE group), Poitiers, France

Full list of author information is available at the end of the article
}

extubation failure [7-10]. In these studies, NIV was applied during the 24-48 hours following extubation and seemed particularly effective in cases of hypercapnia [7-9] while its use appeared pointless in patients at low-risk for extubation failure [11]. Among the six randomized controlled studies of prophylactic NIV, two studies found a reduction in reintubation rate $[7,10]$, whereas the others found no significant difference $[8,9,11,12]$. However, reintubation is the major event independently associated with poor outcome [13], and the main objective of prophylactic NIV should be to avoid reintubation in the most at-risk patients. The studies that have found beneficial effects of NIV in hypercapnic patients included a 
selected population with chronic pulmonary disease admitted in specialized pulmonary units [7-9]. Consequently, more than $30 \%$ of these patients were hypercapnic at the time of extubation $[7,8]$, a finding which may be less prevalent in a general ICU.

We previously determined a subset of patients at highrisk for reintubation, comprising patients older than 65 years and/or having underlying cardiac or respiratory disease [5]. Easily identifiable, these criteria represent a large population that could potentially benefit from post-extubation NIV. Therefore, we aimed to assess the risk of reintubation up to seven days after extubation in patients at high-risk for extubation failure after implementation of a specific prophylactic NIV program.

\section{Methods}

\section{Study design}

This is a prospective before-after study aimed at assessing the effects of a protocol for the routine use of prophylactic NIV after planned extubation in patients at high-risk for reintubation. All patients admitted to the 13-bed medical ICU of our teaching hospital in Créteil and who had undergone planned extubation were prospectively screened. In a first cohort treated from May 2005 to May 2006 (control cohort) we identified a population at high-risk for reintubation as noted in our previously published analysis [5]. After implementing prophylactic NIV following extubation, we prospectively collected data from November 2010 to April 2012 to analyze the risk of extubation failure in these patients and compare it to clinicians' predictions [14]. In the current before-after study we wish to compare the efficacy of this prophylactic NIV protocol. This study was approved by the ethics committee of Henri Mondor hospital (CPP Ile-de-France IX). Signed informed consent was waived because of the observational nature of the study; patients or relatives were nonetheless provided with an informational letter on the aims and conduct of the study.

\section{Weaning protocol}

All ventilated patients were screened every morning by the nurse in charge and a weaning test was systematically performed in those who fulfilled the following weaning criteria: patient awake without continuous infusion of sedatives, $\mathrm{SpO}_{2} \geq 90 \%$ with $\mathrm{FiO}_{2} \leq 40 \%$ and positive end-expiratory pressure (PEEP) $\leq 5 \mathrm{cmH}_{2} \mathrm{O}$, and no need for vasopressors. Failure of the weaning test was defined as the development within 1 hour of any of the following: respiratory rate above 35 breaths $/ \mathrm{min}, \mathrm{SpO}_{2}$ persistently below $90 \%$, heart rate persistently above 130 beats/min, systolic blood pressure below $90 \mathrm{mmHg}$ or above $180 \mathrm{mmHg}$, clinical signs suggesting respiratory distress, profuse sweating, agitation or depressed mental status. When the weaning test was well-tolerated, patients were extubated after 1 hour. In the control cohort the weaning test was performed using a T-piece whereas in the NIV cohort it was performed using a pressure-support (PS) around $7 \mathrm{~cm} \mathrm{H}_{2} \mathrm{O}$ without PEEP. Moreover, a blood gas test was systematically performed at the end of the weaning test in the NIV cohort.

\section{Inclusion criteria}

In the control cohort, we found that patients older than 65 years and those having any underlying cardiac or respiratory disease were at high-risk for extubation failure with a reintubation rate exceeding $20 \%$ in patients who had one of these two factors, and greater than $30 \%$ when the two factors were combined [5]. Therefore, we decided to implement a protocol for the systematic use of prophylactic NIV within the first 24 hours following planned extubation in all patients who fulfilled at least one of these criteria [14]. Patients intubated less than $24 \mathrm{~h}$ and those with a do-not reintubate order were excluded.

Underlying cardiac diseases included left ventricular dysfunction defined by left ventricular ejection fraction $\leq 45 \%$, ischemic heart disease or chronic atrial fibrillation, history of cardiogenic pulmonary edema, or severe valvulopathy. Chronic lung diseases included chronic obstructive pulmonary disease, obesity-hypoventilation syndrome or restrictive pulmonary disease.

\section{Prophylactic NIV protocol}

The study was conducted after the implementation of a nurse-driven NIV protocol to adjust the ventilatory settings and to improve the patient's tolerance to NIV following a simple decision algorithm. NIV was immediately applied after extubation for periods of at least 1 hour, with a minimal duration of 8 hours within the first 24 hours following extubation. NIV was initiated using a PS level of $8 \mathrm{~cm} \mathrm{H} \mathrm{H}_{2} \mathrm{O}$ and a PEEP level of $5 \mathrm{~cm}$ $\mathrm{H}_{2} \mathrm{O}$. The nurse could then gradually adjust the PS level to target a tidal volume at around $6-8 \mathrm{ml} / \mathrm{kg}$ of predicted body weight. An algorithm was used by nurses in case of leaks, which successively included repositioning of the mask, reducing the PEEP level at $2 \mathrm{~cm} \mathrm{H}_{2} \mathrm{O}$, and reducing the PS level by steps of $2 \mathrm{~cm}$ $\mathrm{H}_{2} \mathrm{O}$. NIV was performed via a non-vented full-face mask with an ICU ventilator using a dedicated NIV mode, equipped with a heated humidifier. Ventilator settings, ventilatory parameters, and blood gases were prospectively collected 1 hour after NIV initiation, as well as the number and duration of NIV sessions. Between NIV sessions patients received standard oxygen therapy; high-flow oxygen therapy was never used, either in the control cohort or in the NIV cohort. 


\section{Duration of NIV and criteria for reintubation}

In the absence of acute respiratory failure symptoms 24 hours after planned extubation, NIV was discontinued. If moderate respiratory failure appeared or persisted 24 hours after extubation, prophylactic NIV was continued for periods of 24 hours and reassessed daily until complete disappearance of acute respiratory failure criteria, including: 1) respiratory rate $>25$ / min, 2) clinical signs suggesting increased work of breathing, 3) respiratory acidosis defined as $\mathrm{pH}<7.35$ and $\mathrm{PaCO}_{2}>45 \mathrm{mmHg}$, and 4) $\mathrm{SpO}_{2}<90 \%$ despite $\mathrm{FiO}_{2}$ 0.4.

In the two cohorts, patients were reintubated if they met at least one of the following criteria: 1) clear worsening of respiratory failure with a respiratory rate above 40 breaths per minute, $\mathrm{SpO}_{2}$ persistently below $90 \%$ despite supplemental oxygen, worsening $\mathrm{pH}$ and $\mathrm{PaCO}_{2}$ values with depressed mental status, or persistent inability to remove copious airway secretions; 2) hemodynamic failure (defined as systolic blood pressure $<90 \mathrm{mmHg}$ ) without response to a fluid challenge and need for vasoactive drugs, 3) neurological failure defined as altered consciousness, coma or psychomotor agitation, or 4) cardiac or respiratory arrest.

In the literature, the time interval used to define extubation failure varies between $48[6,15,16]$ and 72 hours $[5,17-19]$, or up to one week $[3,17,20,21]$. Since use of NIV may delay reintubation time [3], we defined extubation failure as the need for reintubation within seven days following extubation.

\section{Statistical analysis}

Continuous variables were expressed as mean \pm standard deviation and compared using Student's $t$-test. Dichotomous variables were expressed as percentage and compared using the Chi-2 test. Our main objective was to compare the rate of extubation failure in the two cohorts using the rate of reintubation $\leq 7$ days as the primary end point. Kaplan-Meier curves were plotted to assess time from extubation to reintubation in patients at highrisk in the two cohorts and compared by the log-rank test. We performed a multivariate analysis using a backward step-down logistic regression model including the non-collinear variables associated with extubation failure with a $\mathrm{p}$ value $<0.15$ using univariate analysis. We considered two-tailed $\mathrm{p}$ values $<0.05$ as significant.

\section{Results}

All in all, 132 patients in the control cohort (12-month period) and 225 patients in the NIV cohort (18-month period) experienced planned extubation (Fig. 1). Sixtyone of the 357 extubated patients $(17 \%)$ needed reintubation at some time in the ICU, of whom $74 \%(n=45)$, $82 \%(\mathrm{n}=50)$ and $97 \%(\mathrm{n}=59)$ were reintubated within the first 48 hours, 72 hours and seven days following extubation, respectively. Acute respiratory failure was the main reason for reintubation $(69 \%, 42 / 61)$. The mortality of patients who needed reintubation reached $51 \%(31 / 61)$.

\section{Patients at high-risk for extubation failure}

In the control cohort, 83 of the 132 extubated patients (63\%) were at high-risk for extubation failure (Fig. 1),

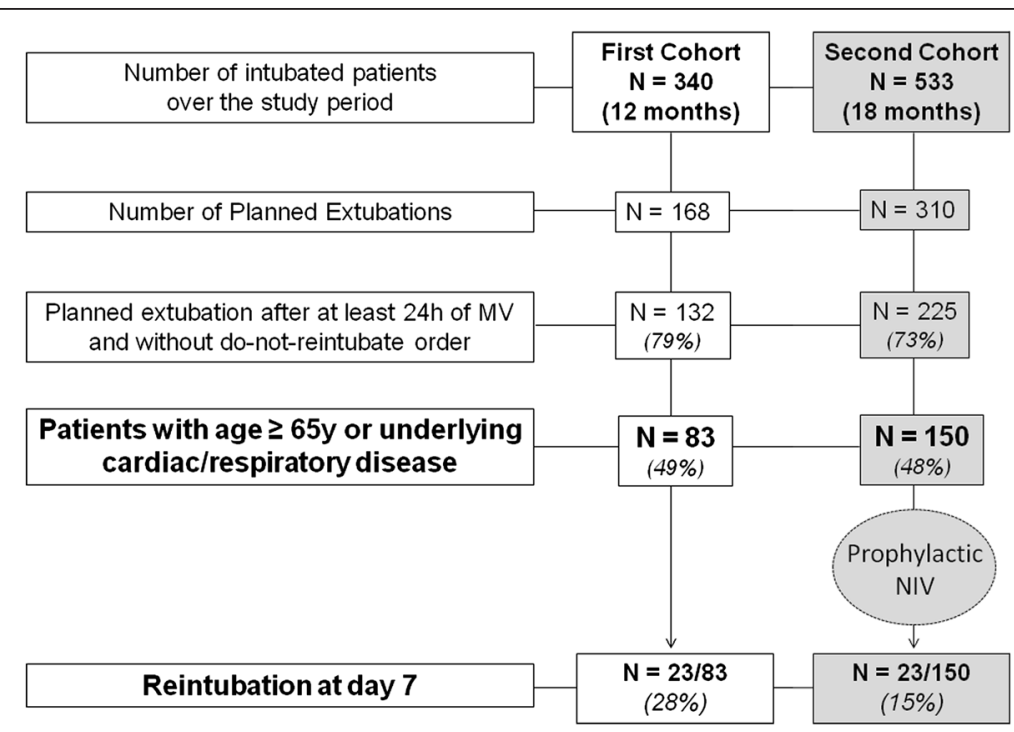

Fig. 1 Flow-chart of the study. All in all, 168 patients in the control cohort and 310 in the noninvasive ventilation (NIV) cohort experienced planned extubation. Patients who were extubated after less than $24 \mathrm{~h}$ of mechanical ventilation (MV) or with a do-not-intubate order were excluded. Among all patients who experienced planned extubation, the proportion of patients at high-risk for extubation failure was similar in the two cohorts: $49 \%$ (83/168) in the control cohort and $48 \%$ (150/310) in the NIV cohort, $p=0.83$ 
and none of them received any prophylactic NIV. In the NIV cohort, 150 of the 225 extubated patients (67\%) were at high-risk and eligible to receive prophylactic NIV. Patients' characteristics did not differ between the two cohorts (Table 1).

All in all, reintubation within seven days following extubation occurred in 46 of the 233 patients considered at high-risk (20\%). This rate was significantly lower in the NIV cohort than in the control cohort, decreasing from $28 \%(23 / 83)$ to $15 \%(23 / 150), \mathrm{p}=0.02$ log-rank test (Fig. 2) whereas the non-at-risk patients did not significantly differ during the two periods: $10.2 \%(5 / 49)$ vs. $10.7 \%(8 / 75), p=0.93$. The rate of reintubation in patients at high-risk treated with NIV was not significantly higher than that in patients at low-risk overall: $15.3 \%$ $(23 / 150)$ vs. $10.5 \%(13 / 124), \mathrm{p}=0.24$.

\section{Use of NIV}

Among the 150 patients considered at high-risk for reintubation in the NIV cohort, 139 (93\%) actually received prophylactic NIV after extubation. In the 11 remaining patients the prophylactic NIV protocol was not followed by the physician or refused by the patient (these patients were kept in the analysis of the NIV cohort performed on an intention-to-treat basis). Given the fact that none of these 11 patients needed reintubation, the rate of extubation failure reached $17 \%$ (23/139) among all patients treated with NIV but remained significantly lower than in patients at high-risk in the control cohort $(\mathrm{p}=0.04$ log-rank test $)$.

The median duration of NIV within the first $24 \mathrm{~h}$ following extubation was $490 \mathrm{~min}$ [interquartile range, $353-559]$ and $54 \%$ of the patients $(75 / 139)$ received more

Table 1 Comparison of the patients at high-risk for reintubation in the 2 cohorts

\begin{tabular}{|c|c|c|c|}
\hline & Control cohort $\mathrm{N}=83$ & NIV cohort $N=150$ & $P$ value \\
\hline \multicolumn{4}{|l|}{ Patients' characteristics } \\
\hline Age, years & $66 \pm 15$ & $69 \pm 11$ & 0.05 \\
\hline Age $\geq 65$ years, $n(\%)$ & $54(65 \%)$ & $109(73 \%)$ & 0.23 \\
\hline Male sex, n (\%) & 49 (59 \%) & $85(57 \%)$ & 0.73 \\
\hline SAPS II at admission, points & $50 \pm 19$ & $49 \pm 18$ & 0.44 \\
\hline Underlying cardiac disease, n (\%) & $45(54 \%)$ & $90(60 \%)$ & 0.39 \\
\hline Underlying chronic lung disease, n (\%) & $31(37 \%)$ & $53(35 \%)$ & 0.76 \\
\hline Reason for intubation & & & 0.81 \\
\hline Acute respiratory failure, $\mathrm{n}(\%)$ & $33(39 \%)$ & $64(43 \%)$ & \\
\hline Shock, n (\%) & $14(17 \%)$ & $29(19 \%)$ & \\
\hline Coma or neurologic disorders, n (\%) & $13(16 \%)$ & $25(17 \%)$ & \\
\hline Postoperative, n (\%) & $20(24 \%)$ & $26(17 \%)$ & \\
\hline Cardiac arrest, n (\%) & $3(4 \%)$ & $6(4 \%)$ & \\
\hline \multicolumn{4}{|l|}{ Variables at time of extubation } \\
\hline SOFA at time of extubation, points & $3.4 \pm 2.2$ & $3.6 \pm 2.3$ & 0.56 \\
\hline $\mathrm{PaO}_{2} / \mathrm{FiO}_{2}$ ratio, $\mathrm{mm} \mathrm{Hg}$ & $283 \pm 95$ & $296 \pm 92$ & 0.30 \\
\hline $\mathrm{pH}$, units & $7.46 \pm 0.06$ & $7.45 \pm 0.05$ & 0.73 \\
\hline $\mathrm{PaCO}_{2}, \mathrm{~mm} \mathrm{Hg}$ & $41 \pm 10$ & $39 \pm 8$ & 0.15 \\
\hline $\mathrm{PaCO}_{2}>45$ mm Hg, n (\%) & $24(29 \%)$ & $27(18 \%)$ & 0.06 \\
\hline Duration of MV prior to extubation, days & $8.0[5.0-13.8]$ & $6.0[4.0-11.8]$ & 0.24 \\
\hline \multicolumn{4}{|l|}{ Outcome } \\
\hline Reintubation < 48 h, n (\%) & $18(22 \%)$ & $16(11 \%)$ & 0.03 \\
\hline Reintubation < 72 h, n (\%) & $20(24 \%)$ & $19(13 \%)$ & 0.02 \\
\hline Reintubation < 7 days, n (\%) & $23(28 \%)$ & $23(15 \%)$ & 0.02 \\
\hline Reintubation at any time in ICU, n (\%) & $24(29 \%)$ & $24(16 \%)$ & 0.02 \\
\hline Total duration of invasive MV, days & $9.0[5.0-16.0]$ & $7.0[4.0-13.8]$ & 0.07 \\
\hline ICU length of stay, days & $14[11.0-22.0]$ & $12.0[7.0-20.0]$ & 0.18 \\
\hline In-ICU mortality, n (\%) & $12(14 \%)$ & $16(11 \%)$ & 0.39 \\
\hline
\end{tabular}

Values are given as mean \pm standard deviation (SD) or as median [interquartile range, from $25^{\text {th }}$ to $75^{\text {th }}$ percentiles]

NIV noninvasive positive pressure ventilation, SAPS /I Simplified Acute Physiology Score II, SOFA Sequential Organ Failure Assessment, MV mechanical ventilation, ICU intensive care unit 


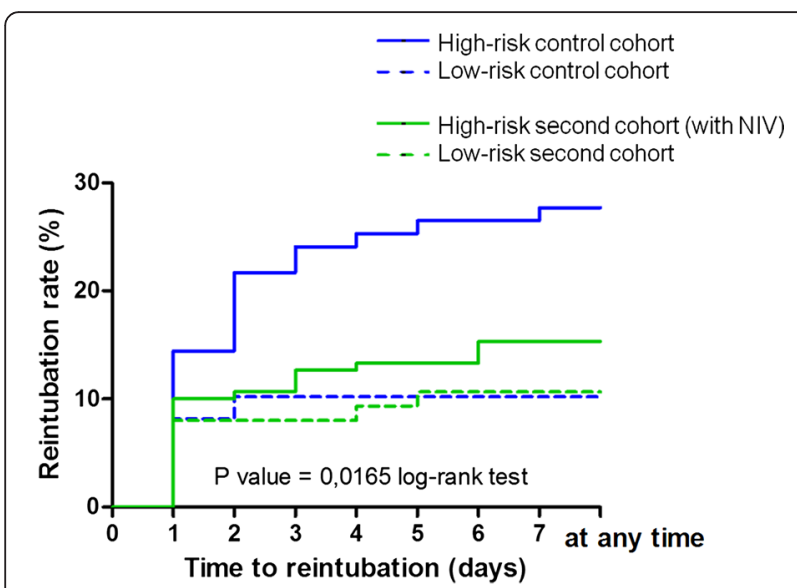

Fig. 2 The Kaplan-Meier plots of the cumulative reintubation rates within seven days following extubation. The rate of extubation failure (reintubation at day 7) significantly differed between the four groups ( $p=0.0165$ log-rank test). Among the patients at high-risk, this rate was significantly lower in the NIV cohort (green solid line) than in the control cohort (blue solid line), decreasing from $28 \%$ (23/83) to $15 \%$ (23/150), $p=0.0225$ by log-rank test. The difference remained significantly lower after having excluded the 11 patients in the NIV cohort who did not effectively receive NIV: $17 \%(23 / 139)$ vs. $28 \%(23 / 89), p=0.0466$ logrank test. By contrast, the rate of extubation failure did not significantly differ during the two periods in patients at low-risk: $10.2 \%$ (5/49) in the control cohort (blue dotted line) vs. $10.7 \%$ (8/75) in the NIV cohort (green dotted line), $\mathrm{p}=0.93$

than eight hours of NIV. NIV was prolonged beyond the first 24 hours in $24 \%$ of the cases $(33 / 139)$ because of persistent respiratory failure with a rate of extubation failure of $21 \%(7 / 33)$.

\section{Factors associated with extubation failure in high-risk patients}

A comparison of patients in cases of success or failure of extubation is given in Table 2. After adjustment using multivariate logistic-regression, patients with a more prolonged duration of mechanical ventilation prior to extubation were more likely to experience extubation failure, whereas the implementation of a prophylactic NIV protocol was independently associated with extubation success (Table 3).

In the NIV cohort, the proportion of hypercapnic patients $\left(\mathrm{PaCO}_{2}>45 \mathrm{mmHg}\right)$ at the end of the weaning test was $16 \%$ overall $(35 / 225)$ and $20 \%$ among the patients at high-risk (30/150).

\section{Discussion}

In this prospective before-after study we assessed the impact of NIV on patient outcome after planned extubation. The implementation of a prophylactic NIV protocol significantly reduced the rate of reintubation in patients identified as at high-risk of extubation failure. Our NIV protocol was applied in a large population of patients at high-risk based on particularly easily identifiable criteria, including those older than 65 years or with underlying cardiac or respiratory disease.

Several studies have suggested that prophylactic NIV could reduce the risk of post-extubation respiratory failure, particularly in hypercapnic patients [7-9]. In these studies, the rate of reintubation assessed within the 48-72 hours following extubation ranged from 19 to $24 \%$ with standard oxygen and from 8 to $11 \%$ with prophylactic NIV [7-9]. While reintubation rates were higher in our population, we defined extubation failure as reintubation up to seven days after extubation. When assessed within the first 48 hours following extubation, our reintubation rates are similar to those reported in previous studies ( $22 \%$ in the control cohort and $11 \%$ in the NIV cohort).

To date, only two studies have found a significant reduction in the rate of reintubation [7, 10]. In the study by Nava and colleagues, prophylactic NIV applied at least eight hours per day during the first 48 hours following extubation significantly reduced the rate of reintubation from $24 \%$ to $8 \%(\mathrm{p}=0.027)$. Another study observed a decrease in reintubation rate from $39 \%$ to only $5 \%$ in the group receiving NIV $(\mathrm{p}=0.016)$ [10]. However, only 38 patients were included in this singlecenter study and the rate of reintubation reported in the control group receiving standard oxygen (39\%) was inordinately high [10].

In the study by Ferrer and colleagues, prophylactic NIV helped to reduce the risk of post-extubation respiratory failure and to decrease mortality, even though the reintubation rate was not significantly decreased [8]. Indeed, as NIV could be used as rescue therapy in case of respiratory distress, it enabled a number of patients from the control group receiving standard oxygen to avoid reintubation. As NIV was chiefly beneficial in hypercapnic patients with chronic respiratory disorders, the same group conducted a second trial including 106 hypercapnic patients [9]. The results confirmed those of the previous study, and use of prophylactic NIV avoided post-extubation respiratory failure [9]. Although 90-day mortality was significantly lower in patients receiving prophylactic NIV, neither in-ICU mortality nor inhospital mortality differed between the two groups, and it would perhaps be premature to attribute the difference in long-term outcome to the use of NIV immediately after extubation. In our study, although we found a significant decrease in the reintubation rate (13\%), ICU mortality did not differ between patients treated with NIV and those receiving standard oxygen.

Whereas NIV seems beneficial in patients considered at high-risk for reintubation, it is probably pointless in patients at low-risk. In a large multicenter trial including nearly 400 patients intubated for more than 48 hours, the use of prophylactic NIV had no impact on outcome 
Table 2 Variables associated with reintubation within the 7 days following extubation among all patients at high-risk for extubation failure

\begin{tabular}{|c|c|c|c|}
\hline & Extubation Success, N = 187 & Reintubation at day $7, \mathrm{~N}=46$ & $P$ value \\
\hline \multicolumn{4}{|l|}{ Patients' characteristics } \\
\hline Age, years & $68 \pm 14$ & $69 \pm 10$ & 0.46 \\
\hline Age $\geq 65$ years, $n(\%)$ & $128(68 \%)$ & $35(76 \%)$ & 0.31 \\
\hline Male sex, n (\%) & $103(55 \%)$ & $31(67 \%)$ & 0.13 \\
\hline SAPS $\|$ at admission, points & $48 \pm 18$ & $53 \pm 19$ & 0.13 \\
\hline Underlying cardiac disease, n (\%) & $107(57 \%)$ & $28(60 \%)$ & 0.65 \\
\hline Underlying chronic lung disease, n (\%) & $69(37 \%)$ & $15(33 \%)$ & 0.59 \\
\hline Use of prophylactic NIV protocol & $127(68 \%)$ & $23(50 \%)$ & 0.02 \\
\hline Reason for intubation & & & 0.40 \\
\hline Acute respiratory failure, $\mathrm{n}(\%)$ & $78(42 \%)$ & $19(41 \%)$ & \\
\hline Shock, n (\%) & $38(20 \%)$ & $5(11 \%)$ & \\
\hline Coma or neurologic disorders, n (\%) & $31(17 \%)$ & $7(15 \%)$ & \\
\hline Postoperative, n (\%) & $34(18 \%)$ & $12(26 \%)$ & \\
\hline Cardiac arrest, n (\%) & $6(3 \%)$ & $3(7 \%)$ & \\
\hline \multicolumn{4}{|l|}{ Variables at time of extubation } \\
\hline SOFA at time of extubation, points & $3.4 \pm 2.2$ & $3.7 \pm 2.7$ & 0.46 \\
\hline $\mathrm{PaO}_{2} / \mathrm{FiO}_{2}$ ratio, $\mathrm{mm} \mathrm{Hg}$ & $289 \pm 90$ & $300 \pm 103$ & 0.47 \\
\hline $\mathrm{PaO}_{2} / \mathrm{FiO}_{2}$ ratio $\leq 200 \mathrm{~mm} \mathrm{Hg}, \mathrm{n}(\%)$ & $30(16 \%)$ & $6(13 \%)$ & 0.61 \\
\hline $\mathrm{pH}$, units & $7.45 \pm 0.06$ & $7.46 \pm 0.06$ & 0.63 \\
\hline $\mathrm{PaCO}_{2}, \mathrm{~mm} \mathrm{Hg}$ & $41 \pm 9$ & $38 \pm 8$ & 0.16 \\
\hline $\mathrm{PaCO}_{2}>45$ mm Hg, n (\%) & $42(23 \%)$ & $9(20 \%)$ & 0.67 \\
\hline Duration of MV prior to extubation, days & $6.0[4.0-11.0]$ & $13.0[5.0-20.0]$ & $<0.001$ \\
\hline Duration of MV prior to extubation > 7 days, n (\%) & 77 (41\%) & $29(63 \%)$ & $<0.01$ \\
\hline
\end{tabular}

Values are given as mean \pm standard deviation (SD) or as median [interquartile range, from 25th to 75th percentiles]

SAPS // Simplified Acute Physiology Score II, NIV noninvasive positive pressure ventilation, SOFA Sequential Organ Failure Assessment, MV mechanical ventilation, ICU intensive care unit

[11]. The patients included in this study were not really at high-risk for reintubation, and the overall reintubation rate did not exceed $10 \%$.

In studies including patients considered at high-risk for extubation failure [7-10], the inclusion criteria were highly heterogeneous and differed from one study to another. Nava and colleagues included patients with hypercapnia (34 \% of the patients) as well as patients with chronic cardiac failure, several comorbidities, weak cough, stridor, or having failed several weaning tests before extubation [7]. Ferrer and colleagues included patients aged $\geq 65$ years or more, a high severity score, or intubated for cardiac failure [8]. The same group subsequently conducted a second trial including only patients with hypercapnia [9]. In another study, prophylactic NIV was used only in patients intubated at least three days for acute respiratory failure [10]. However, in these studies many of the factors constituting inclusion criteria to start prophylactic NIV are not variables clearly associated with reintubation.

From our standpoint, future research efforts should focus on identifying patients at high-risk for reintubation who may benefit from NIV in various ICU settings. On

Table 3 Variables independently associated with reintubation within the 7 days following extubation in patients at high-risk for extubation failure $(\mathrm{N}=233)$

\begin{tabular}{lll}
\hline Multivariate analysis using logistic regression & Adjusted Odds Ratio ${ }^{\mathrm{a}}[95 \% \mathrm{CI}]$ & $\mathrm{P}$ value \\
\hline Duration of MV prior to extubation, per day & $1.09[1.05-1.13]$ & $<0.001$ \\
Use of prophylactic NIV protocol & $0.48[0.24-0.96]$ & 0.04
\end{tabular}

All variables significantly associated with extubation failure with a p value $<0.15$ were included in the model including: male sex, SAPS II, hypercapnia, duration of mechanical ventilation (MV) prior to extubation, and application of prophylactic NIV protocol (NIV cohort)

SAPS /I Simplified Acute Physiology Score II, NIV noninvasive positive pressure ventilation

${ }^{a}$ Values of adjusted odds ratio are taken from the final model including only variables independently associated with extubation failure. Logistic regression was performed using 233 observations and the final model had an area under a Receiver Operating Curve of 0.726 (Hosmer-Lemeshow test 0.683 ) 
the other hand, classification of patients according to the difficulty of their weaning process [22] seems of little help in prediction of extubation failure since the reintubation rate between patients with simple weaning and those with difficult weaning is somewhat similar [4, 23-25]. By contrast, we have found that prolonged duration of mechanical ventilation prior to extubation was a strong predictor of extubation failure, and that patients intubated more than seven days may probably be considered as at high-risk of extubation failure [14]. Since our first study [5], we decided to initiate NIV in a large subset of patients, who were at once particularly easy to identify, and at unacceptably high-risk for reintubation and mortality. Among these patients, only $20 \%$ had hypercapnia during the weaning test and would have received prophylactic NIV in the event that only this criterion had been used.

\section{Limitations}

First, our study was performed in a single center in which our population may have differed from those of other centers in terms of demographic or primary reason for intubation, a factor that limits the generalizability of our results. Although cohort studies obviously do not have the strength of a randomized controlled study, our study was prospective and included the largest number of patients at high-risk treated with prophylactic NIV to date $(n=139)$. The reintubation rate in the non-at-risk patients remained exactly similar between the two periods.

Second, the weaning readiness tests were not performed in the same way in the two cohorts. Low levels of PS were used during the NIV cohort, which may have resulted in lower levels of work of breathing than with T-piece as performed in the first cohort [26]. However, clinical studies have found no difference between the two approaches in terms of extubation outcome [15] and, if anything, the low pressure-support approach could result in a higher extubation rate and potentially more patients in need of reintubation in the NIV cohort.

Third, the data from the NIV cohort were collected four years after the end of the control cohort. Therefore, other changes may have impacted the reintubation rate, such as modification in sedation practice, use of diuretics, special attention paid to patients at highrisk, and our result could be due to an improvement in the process of weaning including prophylactic NIV rather than the implementation of NIV alone. However, the duration of mechanical ventilation prior to extubation was not significantly different between cohorts and the use of the NIV protocol remained independently associated with extubation success, even after adjustment on prior duration of mechanical ventilation. Moreover, the percentage of patients at high-risk was not significantly different between cohorts, thereby suggesting a relatively similar clinical practice.

As a final limitation, the trend toward a lower proportion of hypercapnic patients in the NIV cohort may have partially contributed to the lower reintubation rate compared to the control cohort. However, although it was previously shown that weaning may be more prolonged in hypercapnic patients, it was not significantly associated with extubation failure [4]. In our second cohort, hypercapnia at the end of the weaning test was associated with extubation failure in univariate analysis, but was not an independent predictor of reintubation after adjustment on multiple risk factors [14].

Although our results support the use of NIV rather than standard oxygen in patients at-risk, the beneficial effects found in this study might not be extrapolated to patients treated by high-flow oxygen therapy [27].

\section{Conclusions}

Prophylactic NIV applied immediately after extubation could reduce the reintubation rate in a large population of patients at high-risk for extubation failure including those aged 65 years or older and/or having underlying cardiac or respiratory disease.

\section{Key messages}

- The implementation of a protocol using prophylactic noninvasive ventilation immediately after extubation may reduce the reintubation rate in a large population of patients with easily identified risk factors for extubation failure.

- These patients were older than 65 years and presented with underlying cardiac or respiratory disease.

\section{Competing interests}

The authors declare that they have no competing interests.

\section{Authors' contributions}

AWT has access to all of the data in the study and takes responsibility for the integrity of the data and the accuracy of the data analysis. Conception and design: AWT; Acquisition of data: FB, HBG, KR; Analysis and interpretation of data: $A W T, F B, H B G, K R, A M D, C B B, L B$; Drafting the manuscript or revising it critically for important intellectual content: AWT, FB, HBG, KR, AMD, CBB, and LB. All authors have read and approved the final manuscript.

\section{Funding/Support}

None.

Author details

${ }^{1} \mathrm{CHU}$ de Poitiers, Réanimation Médicale, Poitiers, France. ${ }^{2}$ INSERM CIC 1402 (ALIVE group), Poitiers, France. ${ }^{3}$ Université de Poitiers, Faculté de Médecine, Poitiers, France. ${ }^{4}$ AP-HP, Hôpital Henri Mondor, DHU A-TVB, Service de Réanimation Médicale, CARMAS Research Group, Créteil 94010, France. ${ }^{5}$ Interdepartmental Division of Critical Care Medicine, University of Toronto, Toronto, ON, Canada. ${ }^{K}$ Keenan Research Institute and Department of Critical Care Medicine, St. Michael's Hospital, Toronto, ON, Canada. 
Received: 8 January 2016 Accepted: 11 February 2016

Published online: 26 February 2016

\section{References}

1. Thille AW, Richard J-CM, Brochard L. The decision to extubate in the intensive care unit. Am J Respir Crit Care Med. 2013;187(12):1294-302.

2. Thille AW, Cortés-Puch I, Esteban A. Weaning from the ventilator and extubation in ICU. Curr Opin Crit Care. 2013;19(1):57-64.

3. Girault C, Bubenheim M, Abroug F, Diehl JL, Elatrous S, Beuret P, et al. Noninvasive ventilation and weaning in patients with chronic hypercapnic respiratory failure: a randomized multicenter trial. Am J Respir Crit Care Med. 2011;184(6):672-9

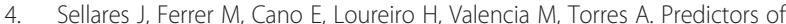
prolonged weaning and survival during ventilator weaning in a respiratory ICU. Intensive Care Med. 2011;37(5):775-84.

5. Thille AW, Harrois A, Schortgen F, Brun-Buisson C, Brochard L. Outcomes of extubation failure in medical intensive care unit patients. Crit Care Med. 2011;39(12):2612-8.

6. Vallverdu I, Calaf N, Subirana M, Net A, Benito S, Mancebo J. Clinical characteristics, respiratory functional parameters, and outcome of a twohour T-piece trial in patients weaning from mechanical ventilation. Am J Respir Crit Care Med. 1998;158(6):1855-62.

7. Nava S, Gregoretti C, Fanfulla F, Squadrone E, Grassi M, Carlucci A, et al. Noninvasive ventilation to prevent respiratory failure after extubation in high-risk patients. Crit Care Med. 2005;33(11):2465-70.

8. Ferrer M, Valencia M, Nicolas JM, Bernadich O, Badia JR, Torres A. Early noninvasive ventilation averts extubation failure in patients at risk: a randomized trial. Am J Respir Crit Care Med. 2006:173(2):164-70.

9. Ferrer M, Sellares J, Valencia M, Carrillo A, Gonzalez G, Badia JR, et al. Noninvasive ventilation after extubation in hypercapnic patients with chronic respiratory disorders: randomised controlled trial. Lancet. 2009;374(9695): 1082-8.

10. Ornico SR, Lobo SM, Sanches HS, Deberaldini M, Tofoli LT, Vidal AM, et al. Noninvasive ventilation immediately after extubation improves weaning outcome after acute respiratory failure: a randomized controlled trial. Crit Care. 2013;17(2):R39.

11. Su CL, Chiang LL, Yang SH, Lin HI, Cheng KC, Huang YC, et al. Preventive use of noninvasive ventilation after extubation: a prospective, multicenter randomized controlled trial. Respir Care. 2012:57(2):204-10.

12. Khilnani GC, Galle AD, Hadda V, Sharma SK. Non-invasive ventilation after extubation in patients with chronic obstructive airways disease: a randomised controlled trial. Anaesth Intensive Care. 2011;39(2):217-23.

13. Frutos-Vivar F, Esteban A, Apezteguia C, Gonzalez M, Arabi Y, Restrepo MI, et al. Outcome of reintubated patients after scheduled extubation. J Crit Care. 2011;26(5):502-9.

14. Thille AW, Boissier F, Ben Ghezala H, Razazi K, Mekontso-Dessap A, Brun-Buisson C. Risk factors for and prediction by caregivers of extubation failure in ICU patients: a prospective study. Crit Care Med. 2015;43(3):613-20.

15. Esteban A, Alia I, Gordo F, Fernandez R, Solsona JF, Vallverdu I, et al. Extubation outcome after spontaneous breathing trials with T-tube or pressure support ventilation. The Spanish Lung Failure Collaborative Group. Am J Respir Crit Care Med. 1997;156(2 Pt 1):459-65.

16. Esteban A, Alia I, Tobin MJ, Gil A, Gordo F, Vallverdu I, et al. Effect of spontaneous breathing trial duration on outcome of attempts to discontinue mechanical ventilation. Spanish Lung Failure Collaborative Group. Am J Respir Crit Care Med. 1999;159(2):512-8.

17. Epstein SK, Ciubotaru RL, Wong JB. Effect of failed extubation on the outcome of mechanical ventilation. Chest. 1997;112(1):186-92.

18. Epstein SK, Ciubotaru RL. Independent effects of etiology of failure and time to reintubation on outcome for patients failing extubation. Am J Respir Crit Care Med. 1998:158(2):489-93.

19. Frutos-Vivar F, Ferguson ND, Esteban A, Epstein SK, Arabi Y, Apezteguia C, et al. Risk factors for extubation failure in patients following a successful spontaneous breathing trial. Chest. 2006;130(6):1664-71.

20. Demling RH, Read T, Lind LJ, Flanagan HL. Incidence and morbidity of extubation failure in surgical intensive care patients. Crit Care Med. 1988; 16(6):573-7.

21. Huang CT, Yu CJ. Conventional weaning parameters do not predict extubation outcome in intubated subjects requiring prolonged mechanical ventilation. Respir Care. 2013;58(8):1307-14.
22. Boles JM, Bion J, Connors A, Herridge M, Marsh B, Melot C, et al. Weaning from mechanical ventilation. Eur Respir J. 2007;29(5):1033-56.

23. Funk GC, Anders S, Breyer MK, Burghuber OC, Edelmann G, Heindl W, et al. Incidence and outcome of weaning from mechanical ventilation according to new categories. Eur Respir J. 2010;35(1):88-94.

24. Tonnelier A, Tonnelier JM, Nowak E, Gut-Gobert C, Prat G, Renault A, et al. Clinical relevance of classification according to weaning difficulty. Respir Care. 2011;56(5):583-90.

25. Peñuelas O, Frutos-Vivar F, Fernandez C, Anzueto A, Epstein SK, Apezteguia $C$, et al. Characteristics and outcomes of ventilated patients according to time to liberation from mechanical ventilation. Am J Respir Crit Care Med. 2011;184(4):430-7

26. Cabello B, Thille AW, Roche-Campo F, Brochard L, Gomez FJ, Mancebo J. Physiological comparison of three spontaneous breathing trials in difficultto-wean patients. Intensive Care Med. 2010;36(7):1171-9.

27. Maggiore SM, Idone FA, Vaschetto R, Festa R, Cataldo A, Antonicelli F, et al. Nasal high-flow versus Venturi mask oxygen therapy after extubation. Effects on oxygenation, comfort, and clinical outcome. Am J Respir Crit Care Med. 2014;190(3):282-8

\section{Submit your next manuscript to BioMed Central and we will help you at every step:}

- We accept pre-submission inquiries

- Our selector tool helps you to find the most relevant journal

- We provide round the clock customer support

- Convenient online submission

- Thorough peer review

- Inclusion in PubMed and all major indexing services

- Maximum visibility for your research

Submit your manuscript at www.biomedcentral.com/submit 